\title{
FRÊNULO DE LÍNGUA ALTERADO E INTERFERÊNCIA NA MASTIGAÇÃO
}

\section{Lingual frenulum alteration and chewing interference}

\author{
Margaret Cross Silva ${ }^{(1)}$, Maria Lúcia Venceslau Carvalho Martins da Costa ${ }^{(2)}$, \\ Kátia Nemr ${ }^{(3)}$, Irene Queiroz Marchesan (4)
}

\begin{abstract}
RESUMO
Objetivo: verificar se indivíduos com alteração do frênulo da língua apresentam alteração na mobilidade da língua e na mastigação. Métodos: a casuística desta pesquisa foi composta por dois grupos, um alvo e outro controle, cada um deles com 10 indivíduos. O grupo alvo apresentava alteração de frênulo da língua. Todos foram submetidos aos protocolos de avaliação de frênulo da língua e de mastigação. As provas de mobilidade da língua e de mastigação foram filmadas. Todos os frênulos da língua foram fotografados. Resultados: os dados coletados mostraram que os indivíduos com alteração do frênulo têm 5,447 vezes mais chance de apresentar alteração na mobilidade da língua que indivíduos com frênulo normal. Os indivíduos com alterações da mobilidade da língua não mantiveram constância quanto ao tempo mastigatório. Quanto à mastigação no Modo de Trituração, 100\% dos indivíduos do grupo controle utilizaram os dentes posteriores. Entre os indivíduos com alteração do frênulo da língua, $47 \%$ mastigaram usando os dentes posteriores e 53\% utilizaram os dentes anteriores e/ou fizeram amassamento com a língua. Com relação as atipias musculares, os indivíduos com alteração de frênulo possuíram 5,714 vezes mais chance de apresentá-las durante a mastigação que os indivíduos do grupo controle. Conclusão: indivíduos com alterações do frênulo da língua apresentaram alterações dos movimentos da língua e alterações na mastigação.
\end{abstract}

DESCRITORES: Freio Lingual; Mastigação; Sistema Estomatognático; Limitação da Mobilidade; Avaliação

\section{INTRODUÇÃO}

As funções orofaciais são realizadas praticamente pelas mesmas estruturas, quando se encontra alteração em uma delas, dificilmente as outras não estão alteradas ${ }^{1-3}$. Sendo assim, o fonoaudiólogo, em sua avaliação deve estar atento a todos os músculos e funções orofaciais, independente da queixa inicial.

(1) Fonoaudióloga; Clínica Fonoaudiológica Cross, São Paulo, SP; Especialista em Motricidade Orofacial.

(2) Fonoaudióloga do Instituto CEFAC, São Paulo, SP; Especialista em Motricidade Orofacial.

(3) Fonoaudióloga; Docente da Universidade de São Paulo, USP, São Paulo, SP; Doutora em Psicologia Social pela Universidade de São Paulo.

(4) Fonoaudióloga; Diretora do CEFAC - Pós-Graduação em Saúde e Educação, São Paulo, SP; Doutora em Educação pela Universidade Estadual de Campinas.

Conflito de interesses: inexistente
Uma das principais funções do sistema estomatognático é a mastigação, para que essa função desenvolva-se com eficiência é importante que todas as estruturas envolvidas nessa atividade estejam íntegras ${ }^{4}$.

A língua é um músculo fundamental para todas as funções orais, incluindo a mastigação. Ela conduz o alimento na cavidade oral, propiciando a mastigação bilateral e alternada ${ }^{1-4}$. O frênulo da língua vai da metade da face inferior da língua (face sublingual) até o assoalho da boca. Suas alterações podem ser relativas à sua fixação, que pode estar anteriorizada ou quanto à sua extensão, quando ele é curto. Destas duas situações pode decorrer uma diminuição da mobilidade da língua ${ }^{5-8}$. Estando a língua com sua mobilidade limitada, as funções que ela exerce podem ser prejudicadas. Existem muitos trabalhos sobre a interferência desta limitação na amamentação e na sucção ${ }^{~}$. O bebê que tenha o frênulo da língua alterado pode ter problemas na pega da mama dificultando a retirada do leite, 
interferindo inclusive no ganho de peso. A liberação cirúrgica do frênulo quando criteriosamente indicada tem promovido uma melhora dessa função ${ }^{8-13}$. Outros artigos discorrem sobre a influência das alterações do frênulo na produção da fala sendo que os sons mais alterados são os líquidos laterais, principalmente o vibrante simples alveolar ${ }^{13-15}$.

$\mathrm{Na}$ clínica fonoaudiológica observa-se que alguns indivíduos, cujo frênulo da língua é alterado, parecem ter a mastigação prejudicada. Distintos trabalhos procuraram conhecer diferentes aspectos da mastigação como tempo da mastigação com diferentes alimentos, eficiência mastigatória, força da musculatura elevadora, dietas alimentares e mastigação ${ }^{16-19}$, porém, não foram encontradas pesquisas estabelecendo relações entre problemas de frênulo e interferência na mastigação. A literatura apenas sugere que indivíduos com alterações do frênulo e ou com dificuldade na mobilidade da língua podem apresentar alteração na mastigação ${ }^{5,7,8,13,20}$. Foi encontrado um único estudo que faz referência a alterações da mastigação em sujeitos com alterações do frênulo da língua ${ }^{21}$.

Sendo assim, o objetivo desse trabalho foi verificar se indivíduos com alteração do frênulo apresentariam alteração na mobilidade da língua e alteração na mastigação, identificando essas alterações.

\section{MÉTODOS}

Estudo transversal em que foram avaliados dois grupos com 10 indivíduos cada um, o grupo alvo e o grupo controle. $\mathrm{O}$ grupo alvo foi composto por indivíduos com alterações do frênulo da língua. Os dois grupos foram avaliados utilizando-se os critérios de avaliação da mobilidade da língua, da mastigação e do frênulo, normal ou alterado, propostos por Marchesan ${ }^{6,7}$. Tanto o grupo alvo, como o grupo controle, foram compostos por pacientes do Instituto CEFAC. A idade variou de 12 anos e 9 meses a 25 anos e 5 meses, com a média de 16,9 anos.

Foram considerados como critérios de exclusão sujeitos que apresentassem: mordida aberta anterior, mordida cruzada uni ou bilateral, Classe III de Angle, ausência de até duas peças dentárias contíguas, respiração oral e ou terem se submetido a frenectomia ou a fonoterapia para reeducação das funções orofaciais.

Os critérios de inclusão foram: indivíduos acima de 12 anos de idade, de qualquer sexo que frequentassem a clínica do Instituto CEFAC.

Todos os indivíduos foram filmados e fotografados durante todas as provas de mobilidade da língua e, apenas filmados durante as provas de mastigação. Os frênulos dos 20 indivíduos também foram fotografados. A câmera utilizada para as fil- magens foi a Panasonic color viewfinder 23 optical zoom lens colocada em um tripé, respeitando-se a distância de 1 metro, e a câmera utilizada para as fotos foi a Lumix Mega O.I.S./28mm Wide.

Para a avaliação da mobilidade da língua solicitaram-se de forma verbal os seguintes movimentos, os quais deveriam ser realizados com a boca aberta: língua para fora e para dentro da boca; língua para cima e para baixo, tocando no lábio correspondente; e língua para as laterais tocando os cantos da boca.

Para a realização da foto do frênulo da língua foi dado o modelo, e solicitado que o indivíduo mantivesse a boca totalmente aberta com a língua para cima, dentro da boca, não tocando em nenhuma estrutura.

Para as provas de mastigação utilizou-se o pão de queijo. Esse alimento foi escolhido por ser consistente, sendo a formação do bolo alimentar bastante coesa. Ele também é de fácil divisão, permitindo obter porções de tamanhos iguais ou muito próximas. Além disso, esse alimento também permite uma boa observação dos movimentos mastigatórios realizados, tanto frontalmente como de perfil. A lateralização do bolo alimentar também é facilmente visualizável. Cada indivíduo comeu 8 metades de pão de queijo. Os sujeitos estavam sentados e foram orientados a colocar na boca, uma metade por vez, antes de iniciar a mastigação.

Para as quatro primeiras porções, a instrução foi a de que, após o alimento ser introduzido na boca, comessem mastigando à vontade. Para as duas primeiras partes, os sujeitos foram filmados de frente. Em seguida, foi solicitado que sentassem de lado, para que pudessem ser filmados de perfil durante a mastigação, sendo que o perfil escolhido sempre foi o direito.

Para os quatro últimos pedaços, os sujeitos foram instruídos a mastigar duas partes do lado esquerdo, sendo uma por vez, e duas do lado direito. Essas quatro últimas ações foram filmadas somente de frente.

Após as avaliações, as filmagens e as fotos foram analisadas separadamente por cinco fonoaudiólogos com experiência de pelo menos sete anos na área de Motricidade Orofacial. Quando havia discordância em algum item, o filme e ou a foto foram revistos para a obtenção de consenso sobre os resultados.

A velocidade com que a mastigação foi realizada foi um dos itens avaliados, que mais gerou divergência entre os avaliadores, uma vez que esse aspecto foi bastante subjetivo. Sendo assim, optou-se pela contagem do número de ciclos mastigatórios em cada porção mastigada, assim como, utilizando-se um cronômetro, contabilizou-se o tempo de cada mastigação. 
A presente pesquisa foi avaliada e aprovada pelo Comitê de Ética em Pesquisa do CEFAC - PósGraduação em Saúde e Educação, sob o n¹32/07, tendo sido considerada como sem risco e com necessidade do consentimento livre e esclarecido.

Os dados obtidos foram submetidos a tratamento estatístico, buscando estabelecer se havia interferência do frênulo alterado, nos movimentos da língua e na função mastigatória. Foram realizadas análise descritiva e análise inferencial, utilizando a técnica de regressão logística e a de regressão para variáveis Gama (modelos lineares generalizados). O nível de significância (p) foi de 5\%.

\section{RESULTADOS}

Quanto à mobilidade da língua encontrou-se que $35 \%$ das pessoas com frênulo alterado ( $\mathrm{N}=$ 21) tiveram a mobilidade alterada, enquanto que apenas $15 \%$ de indivíduos com frênulo normal $(\mathrm{N}=9)$ tiveram alteração na mobilidade. Durante a mastigação, $42 \%$ das pessoas com frênulo normal $(\mathrm{N}=25)$ não apresentaram atipias musculares enquanto nos indivíduos com frênulo alterado, 27\% $(\mathrm{N}=16)$ apresentaram atipias musculares (Tabela 1).

$\mathrm{Na}$ verificação do aspecto trituração durante a mastigação, $100 \%$ das pessoas com frênulo normal fizeram a trituração com os dentes posteriores. Já entre os indivíduos com alteração de frênulo, 47\% $(\mathrm{N}=14)$ apresentaram este modo e $53 \%$ apresentaram outros modos, sendo que: $43 \%(\mathrm{~N}=13)$ utilizaram dentes posteriores e amassamento com a língua; $7 \%(\mathrm{~N}=2)$ utilizaram também os dentes anteriores (incisivos) e 3\% somente os dentes anteriores (Tabela 2).

Observando a variável tempo da mastigação com a mobilidade da língua, vê-se que a mediana no grupo de mobilidade alterada é um pouco superior a do outro grupo (Tabela 3 ).

Para a variável tempo da mastigação, os dois grupos - frênulo normal e frênulo alterado - aparentemente gastaram o mesmo tempo (Tabela 4).

Observando a variável ciclos mastigatórios em cada porção, nos dois grupos de frênulos - alterados e normais - vê-se que os valores da mediana são muito próximos e parece não haver diferenças (Tabela 5).

Analisando a variável mobilidade da língua (Tabela 6), observa-se que a razão de chances para a variável frênulo foi de 5,447 , isto é, uma pessoa que tenha o frênulo alterado tem 5,447 vezes mais chances de ter a mobilidade da língua também alterada ( $p$-valor $=0,003$ ).

Para a variável atipias musculares durante a mastigação (Tabela 7), a razão de chances para a variável frênulo foi de 5,714 , isto é, uma pessoa que tenha o frênulo alterado tem 5,714 vezes mais chances de ter atipias durante a mastigação ( $p$-valor $=0,004)$.

Em relação à variável de ciclos mastigatórios em cada porção (Tabela 8), vê-se que a média para as vezes em que os sujeitos foram instruídas a mastigar da forma como quisessem, foi de 28,57 ciclos/ segundo (igual a 1/0,035). Para as vezes em que foram instruídos a mastigar somente à esquerda ou à direita, por solicitação do fonoaudiólogo, a média foi de 33,3 ciclos/segundo (igual a 1/0,03). Esta diferença de 4,73 foi considerada significante, p-valor $=0,004$.

Não houve diferença significativa entre os dois grupos de padrão mastigatório bilateral ou unilateral, assim como, com relação ao "fechamento labial" durante $\mathrm{o}$ ato de mastigar.

Tabela 1 - Mobilidade da língua e, atipias musculares durante a mastigação, nos indivíduos com frênulos normais e alterados

\begin{tabular}{lcccccccc}
\hline & \multicolumn{4}{c}{ Mobilidade } & \multicolumn{3}{c}{ Atipias musculares } \\
\cline { 2 - 9 } & \multicolumn{2}{c}{ Alterada } & Não alterada & \multicolumn{2}{c}{ Alteradas } & \multicolumn{2}{c}{ Não alteradas } \\
\hline Frênulo & $\mathbf{N}$ & $\%$ & $\mathbf{N}$ & $\%$ & $\mathbf{N}$ & $\%$ & $\mathbf{N}$ & $\%$ \\
\hline Alterado & 21 & 35 & 9 & 15 & 16 & 27 & 14 & 23 \\
Normal & 9 & 15 & 21 & 35 & 5 & 8 & 25 & 42 \\
\hline
\end{tabular}

Tabela 2 - Local de trituração do alimento nos indivíduos com frênulos normais e alterados

\begin{tabular}{ccccc}
\hline & \multicolumn{3}{c}{ Trituração } \\
\hline Frênulo & Posterior & Anterior & Posterior+Língua & Posterior+Língua+Anterior \\
\hline Normal & $30(100 \%)$ & $0(0 \%)$ & $0(0 \%)$ & $0(0 \%)$ \\
Alterado & $14(47 \%)$ & $1(3 \%)$ & $13(43 \%)$ & $2(7 \%)$ \\
\hline
\end{tabular}


Tabela 3 - Tempo de mastigação e mobilidade da língua alterada e normal

\begin{tabular}{cccccccc}
\hline Mobilidade & Mínimo & 10 Quartil & Mediana & 30 Quartil & Máximo & Média & $\begin{array}{c}\text { Desvio } \\
\text { Padrão }\end{array}$ \\
\hline Alteradas & 13 & 20 & 26 & 36 & 63 & 28,37 & 10,95 \\
Não & 11 & 18 & 21 & 27 & 46 & 22,98 & 8,2 \\
Alteradas & 11 & & & & & &
\end{tabular}

Tabela 4 - Tempo de mastigação nos indivíduos com frênulos normais e alterados

\begin{tabular}{cccccccc}
\hline Frênulo & Mínimo & 10 Quartil & Mediana & 30 Quartil & Máximo & Média & $\begin{array}{c}\text { Desvio } \\
\text { Padrão }\end{array}$ \\
\hline Alterado & 11 & 18 & 22 & 31 & 53 & 25,25 & 9,96 \\
Normal & 12 & 19 & 24,5 & 30,25 & 63 & 26 & 10,04 \\
\hline
\end{tabular}

Tabela 5 - Ciclos mastigatórios por porção nos indivíduos com frênulos normais e alterados

\begin{tabular}{cccccccc}
\hline Frênulo & Mínimo & 1o Quartil & Mediana & 3o Quartil & Máximo & Média & $\begin{array}{c}\text { Desvio } \\
\text { Padrão }\end{array}$ \\
\hline Alterado & 12 & 25,75 & 31 & 36,25 & 77 & 32,14 & 11,4 \\
Normal & 15 & 23,75 & 28 & 34 & 71 & 29,92 & 10,12 \\
\hline
\end{tabular}

Tabela 6 - Mobilidade da língua e frênulo

\begin{tabular}{ccccc}
\hline Parâmetro & Estimativa & Erro Padrão & P-valor & IC \\
\hline Frênulo & 5,447 & 0,564 & 0,003 & $(1,866-17,241)$ \\
\hline
\end{tabular}

Tabela 7 - Atipias musculares durante a mastigação e frênulo

\begin{tabular}{ccccc}
\hline Parâmetro & Estimativa & Erro Padrão & P-valor & IC \\
\hline Frênulo & 5,714 & 0,611 & 0,004 & $(1,818-20,659)$ \\
\hline
\end{tabular}

Tabela 8 - Ciclos mastigatórios por porção com mastigação espontânea e dirigida

\begin{tabular}{ccccc}
\hline Parâmetro & Estimativa & Erro Padrão & P-valor & IC \\
\hline Mastigação espontânea & 28,570 & 0,001 & 0,000 & $(27,06-30,27)$ \\
Mastigação dirigida & 33,330 & 0,002 & 0,004 & $(29,48-38,34)$ \\
\hline
\end{tabular}

\section{DISCUSSÃO}

Segundo as pesquisas existentes, os números relativos à incidência de alterações de frênulo variam entre 0,2 a $12 \%$ da população $5,7-15,20,21$. A diferença ou dificuldade em estabelecer estes números está relacionada com a falta de padronização no diagnóstico desta alteração ${ }^{5,7,14}$ e aos poucos estudos sobre a prevalência na população ${ }^{20}$. O número de sujeitos com alterações dentárias, somado ao fato da incidência de alterações de frênulos não ser alta, dificultou a obtenção de um grande número de sujeitos para compor o grupo alvo.

$\mathrm{Na}$ literatura encontram-se dados quanto à 
interferência da alteração de frênulo na mobilidade da língua 7,8,13,20, sendo que a mobilidade alterada é um dos critérios utilizados para indicar possível alteração do frênulo lingual ${ }^{7,14}$. Os achados desta pesquisa concordam com a literatura, a qual aponta que indivíduos com alteração de frênulo possuem maiores chances de apresentarem alterações na mobilidade da língua ${ }^{5,7-15,20}$.

Existem muitos estudos sobre os diferentes aspectos da mastigação na tentativa de compreender, por exemplo, como os músculos funcionam ${ }^{1,17}$, as diferentes fases da mastigação ${ }^{2}$, o tempo de mastigação com diferentes alimentos ${ }^{16}$, de que forma a mastigação ocorre em quem usa próteses dentárias, ou em quem apresenta alterações dentárias, oclusais ou mesmo disfunções da articulação temporomandibular ${ }^{3,22,23}$, ou ainda as diferenças em crianças e adultos ${ }^{4,24}$. No entanto, somente foi encontrado um trabalho, que discorre sobre a possível relação entre a mastigação e as alterações de frênulo ${ }^{21}$. Observa-se que muitos artigos referem que a limitação dos movimentos da língua, possivelmente causada pela interferência do frênulo alterado, possa influenciar de forma negativa no processo da mastigação ${ }^{5,7,8,13,14}$. O trabalho aqui realizado mostrou que, a mobilidade da língua, assim como a mastigação, foi pior nos indivíduos com alteração de frênulo evidenciando que a hipótese inicial dos autores foi confirmada.

$\mathrm{Na}$ literatura encontra-se que o padrão mastigatório deve ser bilateral e alternado, pois este possibilita distribuição da força mastigatória, intercalando períodos de trabalho e repouso musculares e articulares, levando à sincronia e equilíbrio muscular e funcional ${ }^{1-4}$. Estudos demonstram que por vezes há um lado preferencial sem que isso traga prejuízo ao sistema mastigatório ${ }^{17,23,24}$, fato esse comumente presenciado na rotina da clínica. No presente estudo, apesar dos grupos se diferenciarem, por serem ou não portadores de alteração de frênulo, também não foram observadas diferenças significativas entre os grupos quanto ao padrão mastigatório.

A fase de trituração ocorre preferencialmente com os dentes pré-molares e a pulverização em molares ${ }^{25}$. No trabalho realizado por Casas, Kenny e Macmillan ${ }^{24}$, um transdutor foi posicionado no primeiro molar permanente e os autores comprovaram ser este o local onde o alimento é esmagado durante a mastigação. É considerada como parâmetro de normalidade a mastigação realizada com os dentes posteriores (pré-molares e molares) ${ }^{25}$. Os achados do presente estudo mostraram que $100 \%$ dos indivíduos sem alteração de frênulo utilizaram os dentes posteriores na mastigação. Já entre os indivíduos com alteração de frênulo, somente $43 \%$ apresentaram esse padrão, e $57 \%$ utilizaram outros padrões, que envolviam dentes anteriores e/ou amassamento com língua. Tendo em vista que a alteração do frênulo traz limitação ao movimento da língua, pode-se imaginar que a manutenção do alimento entre os dentes posteriores torna-se mais difícil. Sendo assim, o uso do amassamento com a língua e o uso dos dentes situados na porção anterior da cavidade oral possibilitam a execução da mastigação.

Não houve diferença no fechamento labial, durante $\mathrm{o}$ ato de mastigar, entre os dois grupos. No entanto, o grupo com alteração de frênulo apresentou número significativamente maior de atipias musculares durante a mastigação. A atipia mais presente foi a contração exagerada do músculo mentual e dos lábios, que poderia ser justificada pelo maior esforço para manter os lábios fechados, uma vez que esse grupo também apresentou a mastigação mais anteriorizada. Não foram encontrados na literatura achados sobre esta variável.

O que se pode depreender é que quando há algum desequilíbrio no sistema estomatognático, outras forças musculares podem concorrer para alcançar uma adaptação que permita levar a cabo a função pretendida.

A amplitude do ciclo mastigatório bem como o número de ciclos necessários à degradação do alimento depende, além das condições intrínsecas do indivíduo, do tamanho e consistência do alimento. O número de ciclos necessários para preparar o alimento para a deglutição é constante em um mesmo indivíduo para uma mesma porção de um mesmo alimento. Entre diferentes indivíduos esse número é variável, isto é, indivíduos que utilizam um determinado número de ciclos para um tipo de consistência manterão proporcionalmente o número de ciclos para outras consistências. Isto quer dizer que manterão a mesma velocidade, para rápida ou para lenta em todas as situações ${ }^{25,26}$.

Observou-se que nos indivíduos com alteração da mobilidade da língua houve variação quanto ao tempo da mastigação entre as duas primeiras metades do pão trituradas na primeira prova. Esses indivíduos não mantiveram a constância de tempo de velocidade descrita na literatura. A língua, cujo papel é fundamental na mastigação, quando apresenta mobilidade alterada poderá movimentar-se de forma inadequada, provocando essa variação no tempo da mastigação.

Não houve diferença estatisticamente significante entre os dois grupos quanto à amplitude, tempo e número de ciclos mastigatórios. Observouse que nos dois grupos avaliados houve diferença do número de ciclos mastigatórios entre as quatro primeiras porções, mastigadas de forma espontânea, 
quando comparadas com as quatro últimas, as quais foram mastigadas somente à esquerda ou à direita. Na situação dirigida, foi necessário um maior número de ciclos mastigatórios. Isto parece ocorrer por se tratar de um padrão artificial, o qual aconteceu sob instrução e não de forma automática.

Novas pesquisas devem ser desenvolvidas com um número maior de sujeitos. Também deverão ser consideradas as variações de tipo e de grau da alteração do frênulo durante a mastigação.

Como as funções orofaciais interferem no bom desenvolvimento músculo-esquelético, nutricional, emocional e social, e esse trabalho aponta para a possível interferência do frênulo de língua alterado na mastigação, esse é mais um item que deve ser sempre observado nas avaliações de motricidade orofacial.

\section{CONCLUSÃO}

Indivíduos com alteração do frênulo da língua apresentaram mais chances de alteração na mastigação. Os sujeitos com alteração de frênulo apresentaram: maior dificuldade nos movimentos realizados com a língua; modificações no modo de trituração dos alimentos e mais atipias da musculatura perioral durante a mastigação.

\begin{abstract}
Purpose: to check if individuals with lingual frenulum alteration demonstrate chewing interference. Methods: the casuistry of this research was carried out with two groups, a target and a control group, each one with ten individuals. The target group had lingual frenulum alteration. All subjects were submitted to lingual frenulum and chewing evaluation protocols. This procedure was recorded. Results: the collected samples showed that individuals with lingual frenulum alteration have 5.447 times more chances of show tongue mobility alteration than individuals with normal frenulum. Subjects with tongue mobility alteration haven't kept regularity when it comes to mastication period. As for the chewing, on the grinding mode, $100 \%$ of the control group used the back teeth. Among the subjects with frenulum alteration, $47 \%$ used the back teeth and $53 \%$ chewed with the front teeth and/or mashing with the tongue. Regarding muscle atypical movements, individuals with frenulum alteration have 5.714 times more chances to show them during the chewing than individuals from the control group. Conclusion: individuals with lingual frenulum alteration showed tongue mobility alteration and mastication alterations.
\end{abstract}

KEYWORDS: Lingual Frenum; Mastication; Stomatognathic System; Mobility Limitation; Evaluation

\section{REFERÊNCIAS}

1. Muñoz GC, Silva C, Misaki JK, Gomes ICD, Carvalho ARR. Análise dos potenciais elétricos do músculo masseter durante a mastigação de alimentos com rigidez variada. Rev. CEFAC. 2004; 6(2):127-17.

2. Tagliaro ML, Calvi CL, Chiappetta ALML. A fase da incisão no processo da mastigação: enfoque clínico. Rev. CEFAC. 2004; 6(1):24-8.

3. Cavalcanti RVA, Bianchini EMG. Verificação e análise morfofuncional das características da mastigação em usuários de prótese dentária removível. Rev. CEFAC. 2008; 10(4):490-502. dx.doi.org/10.1590/S1516-18462008000400009

4. Lima RMF, Freire OCB, Nepomuceno Filho JL, Stampford S, Cunha DA, Silva HJ. Padrão mastigatório em crianças de 5 a 7 anos: suas relações com o crescimento craniofacial e hábitos alimentares. Rev.CEFAC. 2006; 8(2):205-15.

5. Brito SF, Marchesan IQ, Bosco CM, Carrilho ACA, Rehder MI. Frênulo lingual: classificação e conduta segundo ótica fonoaudiológica, odontológica e otorrinolaringológica. Rev. CEFAC. 2008; 10(3):343-51. dx.doi.org/10.1590/S1516-18462008000300009

6. Marchesan IQ. Avaliação das funções miofuncionais orofaciais. In: Lopes Filho $\mathrm{O}$, Campiotto AR, Levy C, Redondo MC, Bastos WA. Tratado de fonoaudiologia. 2. ed. São Paulo: Tecmedd; 2005. p.713-34.

7. Marchesan IQ. Lingual frenulum: quantitative evaluation proposal. Int J Orofacial Myology. 2005; 31:39-48.

8. Hall DMB, Renfrew MJ. Tongue tie: common problem or old wives' tale. Arch Dis Child. 2005; 90(12):1211-5. 
9. Wallace $H$, Clarke $S$. Tongue tie division in infants with breast feeding difficulties. Int $\mathrm{J}$ Pediatr Otorhinolaryngol. 2006; 70(7):1257-61.

10. Wilson-Clay B. Prejudice against test weighing, like tongue-tie division, should be re-assessed. Arch Dis Child. 2006; 91(12):1042.

11. Breward S. Tongue-tie and breastfeeding: assessing and overcoming the difficulties. Commun Pract. 2006; 79(9):298-9.

12. Dollberg S, Botzer E, Grunis E, Mimouni FB. Immediate nipple pain relief after frenotomy in breastfed infants with ankyloglossia: a randomized, prospective study. J Pediatr Surg. 2006; 41(9):1598-600. 13. Karabulut R, Sönmez K, Türkyilmaz Z, Demirogullari B, Ozen IO, Bagbanci B, et al. Ankyloglossia and effects on breast-feeding, speech problems and mechanical/social issues in children. B-Ent. 2008; 4(2):81-5.

14. Marchesan IQ. Lingual frenulum: classification and speech interference. Int J Orofacial Myology. 2004; 30:31-8.

15. Gonçalves CS, Ferreiro MC. Estudo da relação entre presença de frênulo lingual curto e/ ou anteriorizado e a dorsalização do fone [r] na articulação da fala. Rev CEFAC. 2006; 8(1):56-60.

16. Melo TM, Arrais RD, Genaro KF. Duração da mastigação de alimentos com diferentes consistências. Rev Soc Bras Fonoaudiol. 2006; 11(3):170-4.

17. Oncins MC, Freire RMAC, Marchesan IQ. Mastigação: análise pela eletromiografia e eletrognatografia: seu uso na clínica fonoaudiológica. Rev Dist Comun. 2006; 18(2):155-65.

18. Apolinário RMC, Moraes RB, Motta AR. Mastigação e dietas alimentares para redução de peso. Rev. CEFAC. 2008; 10(2):191-9. dx.doi. org/10.1590/S1516-18462008000200008
19. Felicio CM, Couto GA, Ferreira CLP, Mestriner Júnior W. Confiabilidade da eficiência mastigatória com Beads e correlação com a atividade muscular. Pró-Fono. 2008; 20(4):225-30.

20. Segal LM, Stephenson R, Dawes M, Feldman $P$. Prevalence, diagnosis, and treatment of ankyloglossia: methodologic review. Can Fam Physician. 2007; 53(6):1027-33.

21. Marchesan IQ, Oliveira LR, Costa MLVCM, Araujo RLT. Análise comparativa da mastigação em pacientes com e sem alteração do frênulo lingual. In: XV Congresso Brasileiro de Fonoaudiologia e VII Congresso Internacional de Fonoaudiologia; Gramado, RS, 16 a 20 de out 2007.

22. Pena CR, Pereira MB, Bianchini EMG. Características do tipo de alimentação e da fala de crianças com e sem apinhamento dentário. Rev. CEFAC. 2008; 10(1):58-67. dx.doi.org/10.1590/ S1516-18462008000100009

23. Souza DR, Salvat RP, Bianchini EMG, Ferreira VJA. Características mastigatórias em portadores de disfunção temporomandibular: estudo comparativo. Rev Soc Bras Fonoaudiol. 2005; 10(3):55-60.

24. Casas MJ, Kenny DJ, Macmillan RE. Buccal and lingual activity during mastication and swallowing in typical adults. J Oral Rehabil. 2003; 30(1):9-16.

25. van der Bilt A, Engelen L, Pereira LJ, van der Glas HW, Abbink JH. Oral physiology and mastication. Physiol Behav. 2006; 89(1):22-7.

26. Woda A, Foster K, Mishellany A, Peyron MA. Adaptation of healthy mastication to factors pertaining to the individual or to the food. Physiol Behav. 2006; 89(1):28-35.
RECEBIDO EM: 11/04/2009

ACEITO EM: 29/09/2009

Endereço para correspondência:

Margaret Cross Silva

Rua Desembargador Ferreira França, 40 ap. 122 B

São Paulo - SP

CEP: 05446-050

E-mail: mcross@uol.com.br 